\title{
Calibration of Panoramic Catadioptric Sensors Made Easier
}

\author{
Jonathan Fabrizio ${ }^{1} \quad$ Jean-Philippe Tarel $^{2} \quad$ Ryad Benosman $^{1}$ \\ ${ }^{1}$ UPMC, LISIF, 4 place Jussieu \\ 75252 Paris cedex 05, box 164, France \\ ${ }^{2}$ LIVIC (INRETS-LCPC), 13 Route de la Minière \\ 78000 Versailles, France
}

\begin{abstract}
We present a new method to calibrate panoramic catadioptric sensors. While many methods exist for planar cameras, it is not the case for panoramic catadioptric sensors. The aim of the proposed calibration is not to estimate the mirror surface parameters which can be known very accurately, but to estimate the intrinsic parameters of the CCD camera and the pose parameters of the CCD camera with respect to the mirror. Unless a telecentric lens is used, this pose must be estimated, particularly for sensors that have a unique effective view point. The developed method is based on the original and simple idea that the mirror external and internal boundaries can be used as a 3D calibration pattern. The improvement introduced by our approach is demonstrated on synthetic experiments with incorrectly aligned sensors and validation tests on real images are described. The proposed technique opens new ways for better designed catadioptric sensors where self-calibration can be easily performed in real-time in a completely autonomous way. In particular this should allow to avoid errors due to vibrations one can notice when using catadioptric sensors in practical situations.
\end{abstract}

\section{Introduction}

Panoramic sensors have become essential in mobile robot applications. Indeed, catadioptric sensors are by far the fastest and easiest way to get $360^{\circ}$ field of view. This feature of panoramic sensors is also attractive for a wide range of applications, such as surveillance and teleconferencing.

There is a lot of prior work on planar camera calibration. The two main streams can be described as using a calibration pattern and self-calibration, i.e using features extracted in the images without a priori knowledge on the 3D. If the need of camera calibration is clearly understood for a planar camera, it is not always the case with a catadioptric sensor where its specifications are often too much trusted. One reason is that an incorrectly aligned catadioptric sensor is a non-single viewpoint optical system, and thus it is needed to introduce caustics, as explained in [10]. A catadioptric sensor is generally composed of two aligned parts, one is the catadioptric mirror and the other is a CCD camera. If it is reasonable to trust the specification used for the optical manufacturing of the mirror, it is much more difficult to accurately align the CCD camera with respect to the mirror and to merge the focus point of the mirror with the focus point of the CCD camera. Thus at a minimum, we must estimate both the intrinsic parameters and the pose parameters of the CCD camera with respect to the mirror for any catadioptric sensor. Fig. 7 simulates the effect of direct use of the specifications of a the catadioptric sensor, when the focus point of the mirror and of the CCD camera are not merged.

Previous works on the calibration of catadioptric cameras are not numerous, and primarily focus on particular mirror types. For conic mirrors, a technique [2] using a calibration pattern mounted all around the sensor was proposed. This kind of calibration technique can be easily extended to all kinds of catadioptric sensors [9]. In [4, 6], the authors treat the case of a parabolic mirror. The technique proposed in [4] is based on the interesting property that a parabolic mirror can be calibrated without any knowledge on distances in the scene, only three parallel lines are required. However this property does not apply to non-parabolic mirrors. Thus the proposed technique cannot be easily generalized to all kinds of catadioptric sensors.

Contrary to these, a method in [6] requires no calibration pattern. Here, two techniques are proposed for parabolic mirrors. The first one, the so-called circle-based self calibration, uses only the bounding circle in one image. This technique is simple to implement and does not need any knowledge of the scene or extraction of features in the scene. The second proposed technique is based on points tracked across an image sequence. If no calibration pattern is required, the accuracy of the result could suffer in case of tracking difficulties and of small number of feature points. 
We propose a calibration method which can be seen as a generalization to all kinds of catadioptric mirrors of the circle-based self calibration. The idea is to assume the surface parameters of the mirror are known and to use the boundaries of the mirror as a calibration pattern. Like in the circle-based self calibration, the external border of the panoramic image is fitted, but also the internal one, i.e the border of the area where the camera is seen. As a consequence, the proposed method does not require any special calibration pattern in the scene, it uses only one image, and can be easily applied to all kind of catadioptric sensors without telecentric lens. Our method takes advantages of the use of a calibration pattern without having its restrictions, since the $3 \mathrm{D}$ calibration pattern is the mirror itself. The algorithm relies on two homographic mappings between mirror border points and their images as described in [7, 5].

The paper is organized as follows: in the first section, the sensor geometry is recalled, and the proposed calibration method is explained. Then, the efficiency of the proposed calibration method is experimentally demonstrated on synthetic images. Tests are also performed to validate the proposed method on real images.

\section{Calibration Method}

\subsection{Geometry of the Sensor}

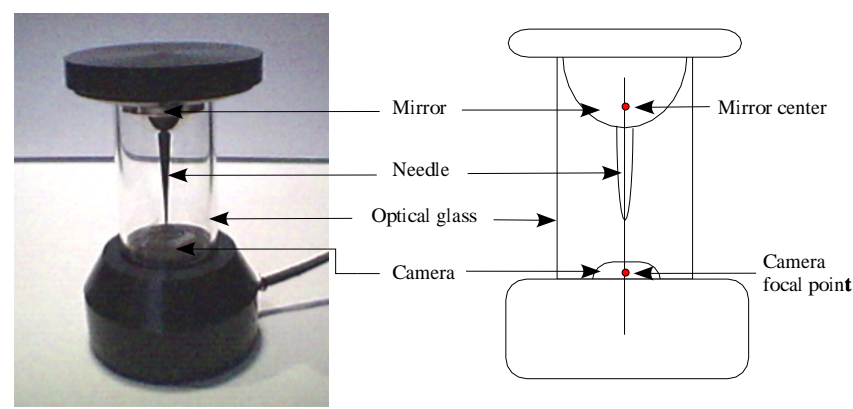

Figure 1. The catadioptric sensor: a planar camera is assembled with a mirror, at the bottom of the mirror a black needle is mounted.

The sensor used is developed by Vstone [3] (as shown in Fig. 1). It is composed of a NTSC color camera and a hyperbolic mirror. The profile and the shape of the mirror are known based on the sensor data sheet. This is not an assumption, the profile and shape of the mirror have to be perfectly known, this is provided by the manufacturing progress that guarantees a precision of less than one micron.
In case of manufacturing errors in the mirror profile, projection errors will prevent the mirror from any use. The used sensor has the particular property that, at the bottom of the mirror, a little black needle is mounted to avoid unexpected reflections.

This kind of camera respects the fixed viewpoint constraint [1]. This property allows the generation of geometrically correct perspective images and simplifies the computation of incoming rays reflected by the mirror. Setting the camera's focal point exactly on the second focus point of the hyperbola simplifies the computation of all reflection of intersecting rays. Without this simplification, the computation would have relied on the determination of all normal vectors of the mirror. If the camera and mirror are incorrectly aligned then multiple viewpoints are obtained and caustics must be considered [10]. This explains the importance of the estimating of the pose of the mirror with respect to the camera focal point.

\subsection{Calibration Principle}

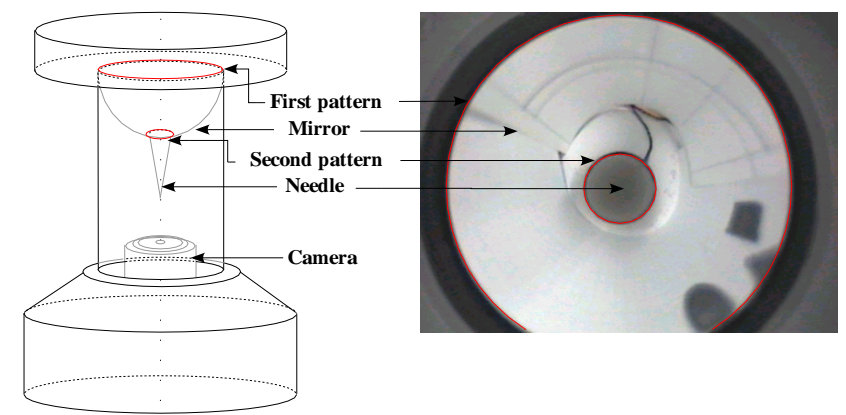

Figure 2. Each of the circles appearing in the image corresponds to a known section of the mirror. The specifications of the mirror being perfectly known, we can assign to each of the sections a known radius and center. Large circle $C_{1}$ corresponds to the upper edge of the mirror, while smallest one $C_{2}$ is due to the intersection of the mirror and the black needle.

The calibration method is based on the principle used for planar cameras in $[7,5]$. In this technique, two different 3D planes are required. The calibration method $[7,5]$ estimates an homography (or projective transform) $H_{1}$ between the image plane and the first plane $P_{1}$. This is usually done using points with known positions in $P_{1}$. The $2 \mathrm{D}$ position in the image of these 3D points is thus also required. With the same manner, an homography $\mathrm{H}_{2}$ can be computed between the image plane and the plane $P_{2}$. The question is then: 
how to compute these 3D points on the mirror, and their 2D images?

As shown in Fig. 2, the mirror boundaries are two perfect circles: the edge of the upper mirror circle, and the edge of the intersection of the black needle with the mirror. These two circles named $C_{1}$ and $C_{2}$ are lying on two parallel planes $P_{1}$ and $P_{2}$. Indeed, each circle corresponds to a planar section of the mirror. The shape of the mirror being perfectly known, the center and radius values of circles $C_{1}$ and $C_{2}$ can be computed from the specifications of the mirror, or easily measured from the sensor. These two circles are projected as ellipses in the image due to the fact that the CCD pixels are not squares and the CCD camera and the mirror are not correctly aligned. These ellipses are named $E_{1}$ and $E_{2}$.

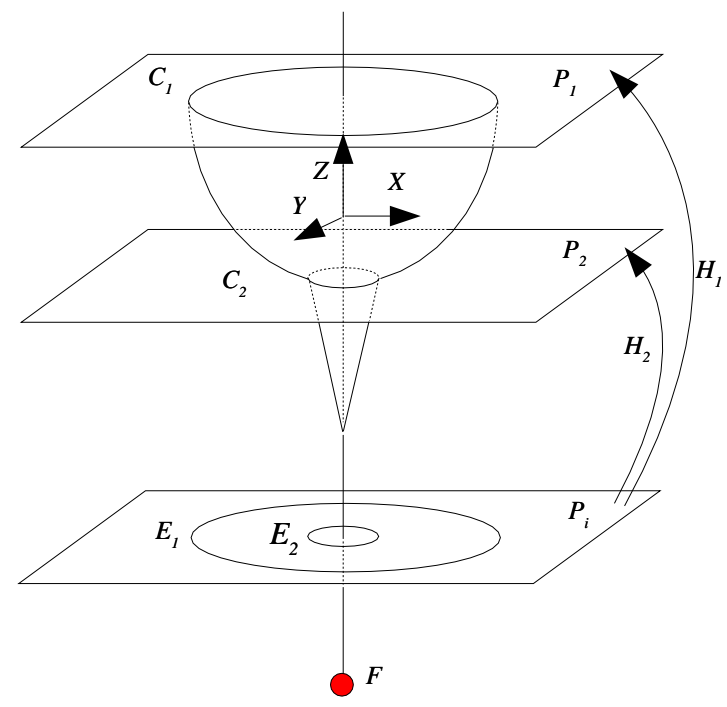

Figure 3. Computation of homographies $\mathrm{H}_{1}$ and $\mathrm{H}_{2}$ between the image and planes $P_{1}$ and $P_{2}$.

The idea is thus to use $C_{1}, C_{2}, E_{1}$, and $E_{2}$ for computing homographies $H_{1}$ and $H_{2}$. More precisely, we compute an homography $H_{1}$ between the image plane containing ellipse $E_{1}$ and the plane $P_{1}$ containing the upper circle $C_{1}$. And with the same manner, we compute an homography $\mathrm{H}_{2}$ between the image plane containing ellipse $E_{2}$ and the plane $P_{2}$ containing the lower circle $C_{2}$ as illustrated by Fig. 3 .

However, it is well known that it is not possible to estimate a unique homography between two corresponding conics lying on two different planes. To overcome this difficulty, we sample each conic according to a regular angular step. For each angular step, we extract the corresponding ellipse points $e_{1 i}$ for $E_{1}$ and $e_{2 i}$ for $E_{2}$. We then apply the same angular sampling technique to circles $C_{1}$ and $C_{2}$ to generate scene points $c_{1 i}$ and $c_{2 i}$ respectively. These four sets of points give us all the information required by the calibration method [7,5], since we can assign, to each image point a known 3D point lying on two different known parallel planes. Therefore, by applying [7, 5], we can easily compute $H_{1}$ from $e_{1 i}$ and $c_{1 i}$, and $H_{2}$ from $e_{2 i}$ and $c_{2 i}$.

\subsection{Image Feature Extraction}

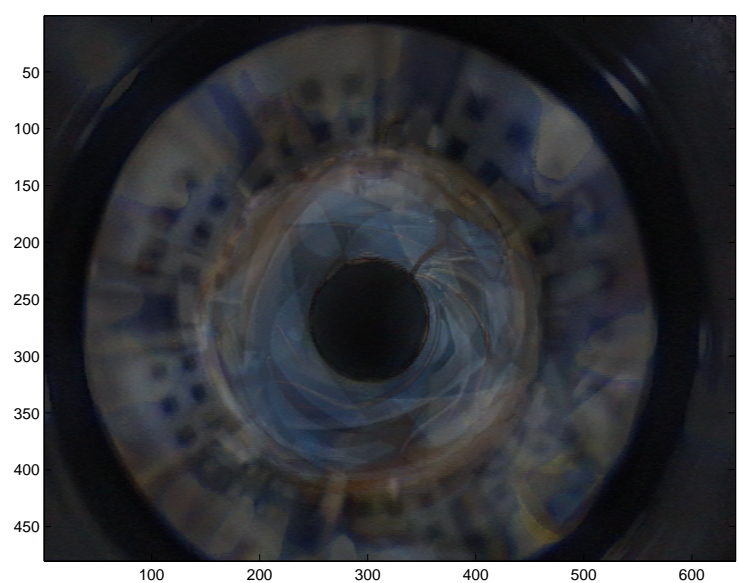

(a)

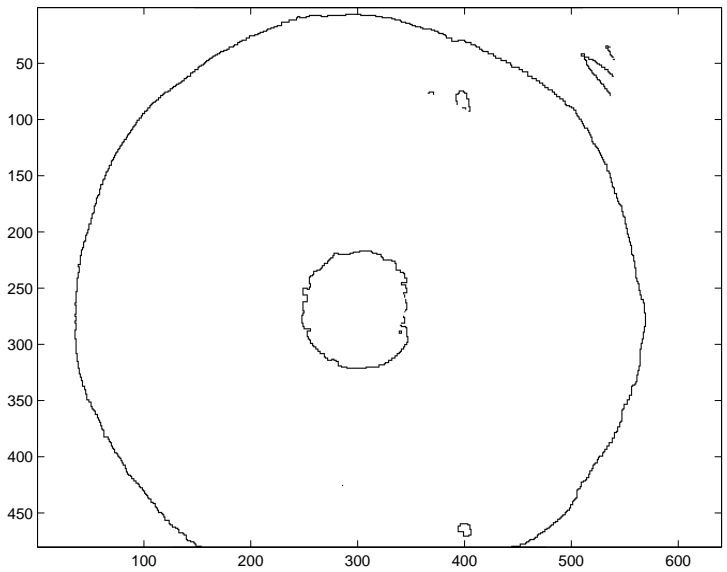

(b)

Figure 4. Image of the intensity variances on $14 \mathrm{im}$ ages obtained by two by two subtraction (a), and its edges obtained using mathematic morphology $(b)$.

In order to compute $H_{1}$ and $H_{2}$, ellipses $E_{1}$ and $E_{2}$ need to be extracted from the image. When only one image is given, several techniques can be applied such as an extended hough transform, constrained snakes, robust fitting of ellipses. We choose to consider the case where a few images of an unknown scene are given. This leads to an easier implementation technique well adapted to mobile robotics 
applications. Indeed, the proposed method was initially developed in the context of an intelligent transportation system where the catadioptric sensor is used for localization purposes mounted on the top of a vehicle.

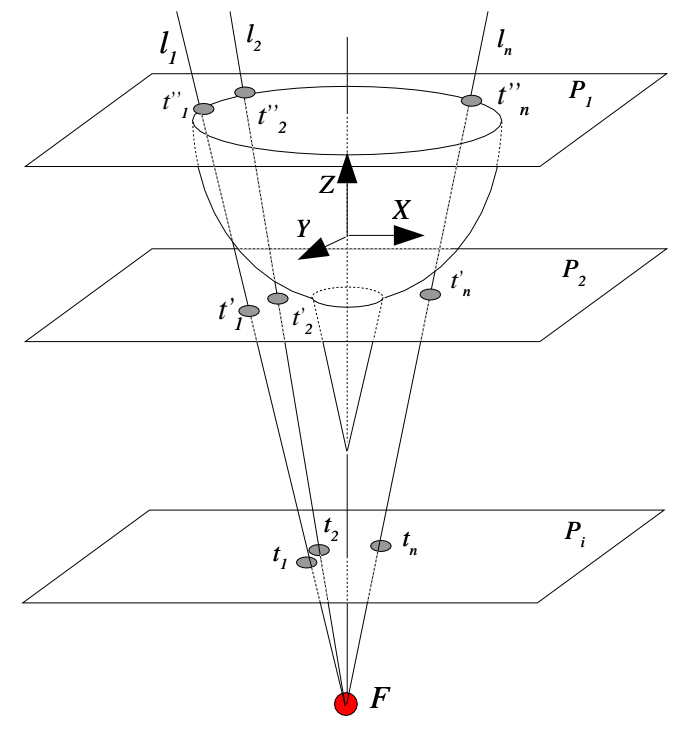

Figure 5. The determination of the focal point: All lines defined by couple $\left(t_{i}^{\prime}, t_{i}^{\prime \prime}\right)$ converge on the focal point of the camera.

The ellipse extraction method is simply based on detecting the image regions corresponding to the mirror and then on fitting ellipses as its internal and external boundaries. Indeed, the persistently present black pixels in the image correspond to the black mounting device of the mirror and of the black needle. From one image to another, pixels with varying values are proportion of incoming light from what is seen in the scene. By weighting those proportion over time, we can separate the three regions (mounting device, mirror, needle) by detecting the region in the image where intensities are varying a lot with respect to the others. For instance, this can be simply performed by taking a sequence of successive images, subtracting them two by two, and make the average of all results. The average variances of each pixel is estimated from the image sequence, as illustrated in Fig. 4(a).

After this processing, all needle and mounting device pixels have a low variance, and contrariwise, the scene pixels will have large variance. These three regions are then separated by a simple thresholding and boundaries computed using any kind of edge detector. For instance, we have used mathematical morphology as shown in Fig. 4(b). Then by applying ellipse fitting technique [8] on the edges, the two ellipses parameters can be estimated. The advan- tage of this technique compared to more classical conic fitting, is that no hyperbola can be obtained. When the sensor is mounted on a mobile robot, this method can be simply applied by the robot under rotation.

\subsection{Pose Estimation}

In order to compute the focus point of the CCD camera, we follow the scheme described in [7]. We choose a set of $n$ points $t_{i}$ in the image, as shown in Fig. 5. Then, every point $t_{i}$ is back-projected onto each plane $P_{1}$ and $P_{2}$, in points $t_{i}^{\prime}$ and $t_{i}^{\prime \prime}$, using homographies $H_{1}$ and $H_{2}$ respectively. Each couple $\left(t_{i}^{\prime}, t_{i}^{\prime \prime}\right)$ determines a line $l_{i}$ in space. All these lines converge in one point $F$ corresponding to the focus point of the camera. Notice that points coordinates are expressed in the mirror coordinate reference system $R$, centered on one of the focus point of the mirror, and aligned with the $z$ axis.

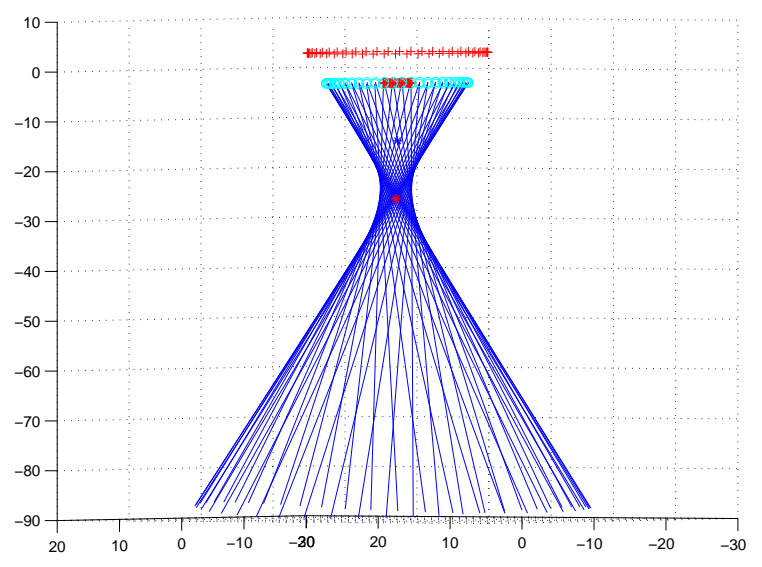

Figure 6. Side view of the $3 D$ representation of the calibrated catadioptric sensor: on top two segments corresponding to the mirror calibration points, the lines correspond to the line of sight of corresponding image points, their intersection is the camera focal point.

We still have to estimate the CCD camera orientation. We again follow [7], but we found out that the proposed technique is not always reliable, and we thus propose an improved method. We have a set $t_{i}$ of $2 \mathrm{D}$ points in the image, and their corresponding 3D points in space $t_{i}^{\prime}$ in homogeneous coordinates. The reference system is now set to $F$ which is now known. As noticed in [7], the computation of the CCD camera orientation consists in minimizing the least square error:

$$
\sum_{i}\left\|t_{i}-N t_{i}^{\prime}\right\|^{2}
$$


where $N$ is the $2 \times 4$ matrix coding the CCD camera orientation, i.e the matrix of the camera base vectors corresponding to the image. This least square problem is solved as a linear system. However, the obtained solution is not reliable since the constraint of orthogonal image axis is not enforced. Let the sets $N_{1}$ and $N_{2}$ be the two lines of $N$, then the constraint of orthogonal axis can be written as $N_{1}^{t} N_{2}=0$. This constraint is quadratic and thus can be easily introduced in the minimization problem using Lagrange multipliers. The minimization now becomes:

$$
\sum_{i}\left\|t_{i}-N t_{i}^{\prime}\right\|^{2}+\lambda N_{1}^{t} N_{2}
$$

where $\lambda$ is set a priori to a small value. The solution is still obtained solving a linear system.

As a consequence, the pose between the mirror and the CCD camera is thus estimated, with the intrinsic parameters of the CCD camera.

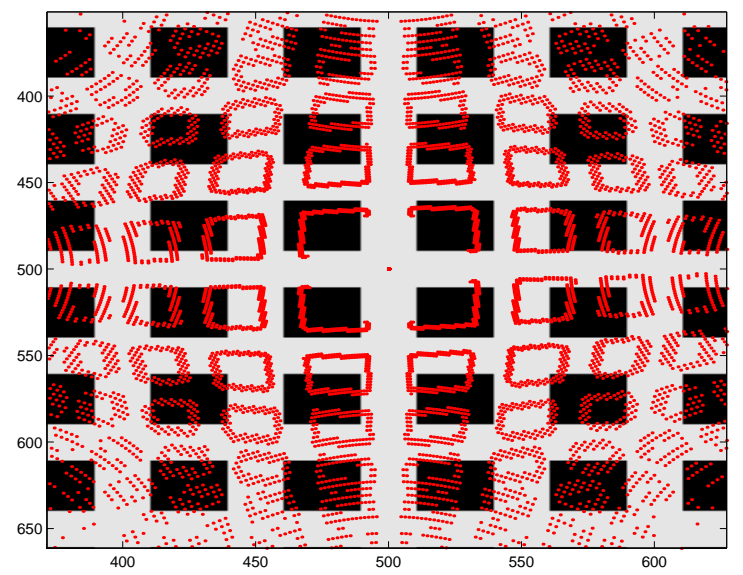

Figure 7. The original scene is made of black rectangles. The image with an incorrectly aligned catadioptric sensor is simulated (CCD focused point moved of $5 \mathrm{~mm}$ along the optical axis from the mirror focus point). The edges of this image are back projected on the true scene assuming that the camera is perfectly aligned. These edges badly superimposed with the original edges.

\section{Validation}

The proposed calibration method can suffer from a lack of precision from image feature extraction. Due to the nonlinearity involved in the projection process for catadioptric cameras, it is difficult to obtain analytical equation relating error in the image to error in the calibration parameters.
Therefore, we have studied the accuracy of calibration on a synthetic catadioptric sensor, eventually incorrectly aligned, making synthetic images. Notice, that the results obtained with these synthetic experiments are less generic than formal derivations since they depend on the shape of the used mirror.

\subsection{Feature Detection Errors}

A key question for our method is to know how sensitive it is to errors in the feature detection, i.e errors in fitted ellipse parameters. Indeed, the accuracy on the parameters of $C_{2}$ is relatively poor, since $C_{2}$ can be seen as a relatively small ellipse in the image. To study this problem, we check the calibration method and its accuracy on a synthetic but realistic sensor. We use the exact profile of our mirror and a calibration matrix close to the specifications given by the CCD manufacturer. To simulate errors, we projected the ideal mirror boundaries onto a virtual image using the ideal matrix of calibration, we also add perturbations on the obtained ellipse parameters. For every perturbation, new calibration parameters are estimated.

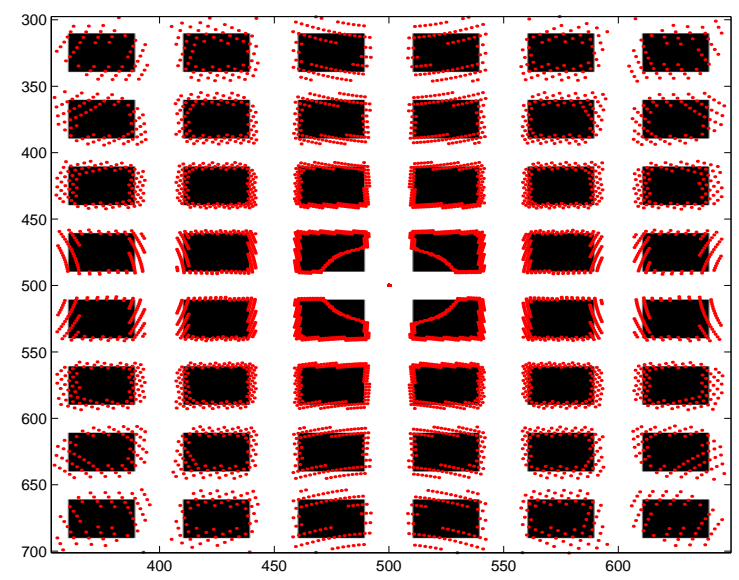

Figure 8. The original scene is made of black rectangles. The image with an incorrectly aligned catadioptric sensor is simulated (CCD focus point moved of $5 \mathrm{~mm}$ along the optical axis from the mirror focus point. The edges of this image are back-projected on the true scene using the estimated calibration parameters. These edges well superimposed with the original edges, contrary to the case where aligned sensor is assumed as shown in Fig 7.

Five parameters describe an ellipse, however we treat variations on only four: scalings on one axis, and translations of the center. These four parameters (for both ellipses) were perturbed independently. It appears that parameters 
which introduces the highest errors in the estimation of the pose parameters, is the axis lengths of the small ellipse. We first noticed that when any of the four parameters of the large ellipse $E_{1}$ is $2 \%$ perturbed, the positioning errors introduced in the estimation of the camera's position is around $1.3 \mathrm{~mm}$. Similarly, when any of the four parameters of the small ellipse $E_{2}$ is $2 \%$ perturbed, the error on the pose is around $1.0 \mathrm{~mm}$. A $2 \%$ perturbation means 4 to 5 pixels for all the parameters unless it is the axis lengths of the small ellipse $E_{2}$. Indeed, if we reduce the axis lengths by $2 \%$ for $E_{2}$ its implies only about 1 pixel in the image. Nevertheless an error on $1 \mathrm{~mm}$ in pose is not too bad for a 1 pixel feature detection accuracy. We also noticed that the errors implied by small perturbations of the ellipse center as on axis lengths are linearly related to the errors on the focal point $F$.

\subsection{Pose Errors}

In order to test the proposed calibration method, we used a synthetic calibration grid composed of squares and set the virtual catadioptric sensor at a known height. We misaligned the virtual CCD camera $5 \mathrm{~mm}$ along the $z$ axis from its optimal position (i.e focus point of the mirror with perfectly aligned sensor) and generated the corresponding image of the calibration pattern. We then back-projected the image edges assuming that the optical center of the camera is at its optimal position. The result is illustrated by Fig. 7, where we can clearly see that the projection does not fit with the calibration pattern geometry.

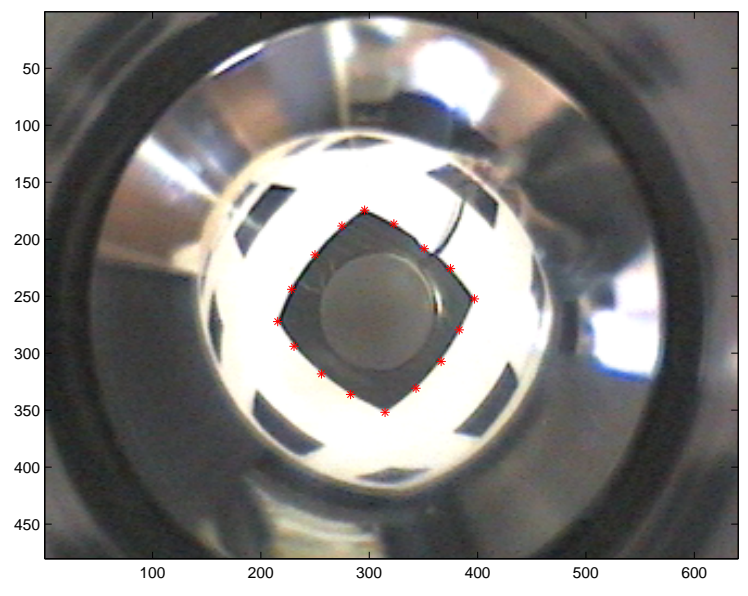

Figure 9. Testing the calibration method on real images : The catadioptric sensor is mounted on top of a flat calibration pattern at a known height.

We applied the proposed calibration method on the synthetic catadioptric image and then, using the estimated pose of the mirror with respect to the CCD camera, we back projected the image edges on the true scene, as illustrated in Fig. 8. To be consistent with the non-unique view point model, the back-projection is computed applying the reflection laws using the normal vector to the surface of the mirror. As displayed in Fig. 8, the edges perfectly match with the original squares edges. This means that the calibration method accurately corrects the misalignment between the camera and mirror focus points. In our synthetic experiments, such an accurate correction can be obtained for a misalignment in a range from $-5 \mathrm{~mm}$ to $5 \mathrm{~mm}$. Larger values are quite unrealistic.

Another advantage of the proposed calibration method is that it can also check if the single view-point assumption is valid or not. This can be easily done by comparing the position of the camera focus point with the position of the mirror focus points obtained from the mirror surface specifications. In case of a not valid single view-point assumption, the classical reflection laws are applied.

\subsection{Real Experiment}

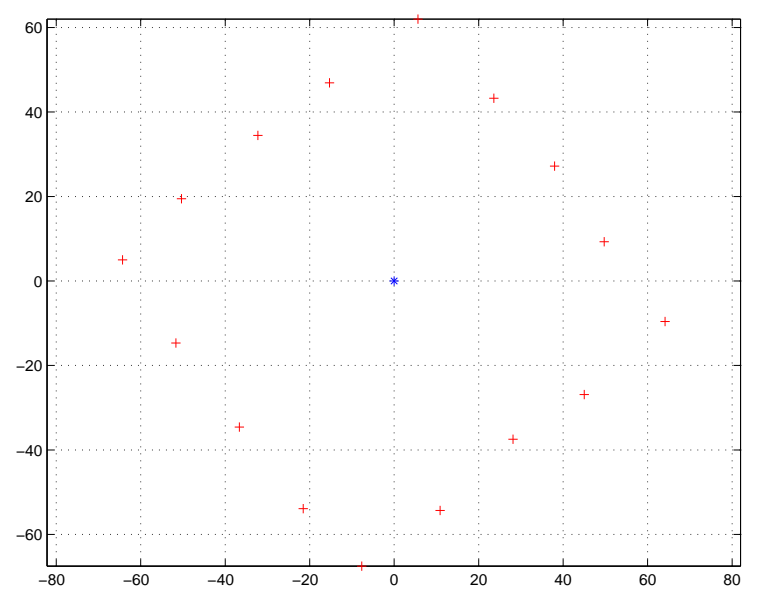

Figure 10. Simple check of the obtained calibration, on a real image, using a squared known shape. The back-projection on the ground of points selected in Fig. 9 is displayed.

To show the validity of the proposed calibration technique, we applied it on real images. The sensor is set above a flat calibration pattern composed of squares with known metrics as shown by Fig. 9 .

The calibration is performed, the resulting $F$ estimation is shown in Fig. 6. This figure shows a side view of the calibrated sensor. On top, two segments can be seen, corresponding to the used calibration points chosen on each mirror circles $C_{1}$ and $C_{2}$. The lines represent the camera 
line of view of each image calibration point used. Their intersection corresponds to the camera focal point.

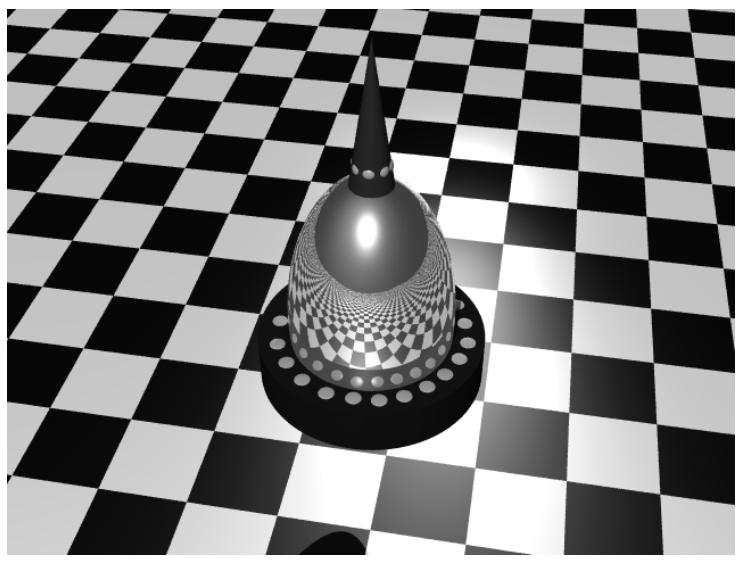

Figure 11. Example of new mirror design for improved catadioptric sensors with a black needle.

To show the validity of the obtained calibration on the real catadioptric sensor, a squared pattern is positioned under our sensor. Using the retrieved projection parameters, we back-project the rays of sight of a few image points along the squared pattern, on the ground plane. As shown in Fig. 10, the result is a square, where square angles are correct.

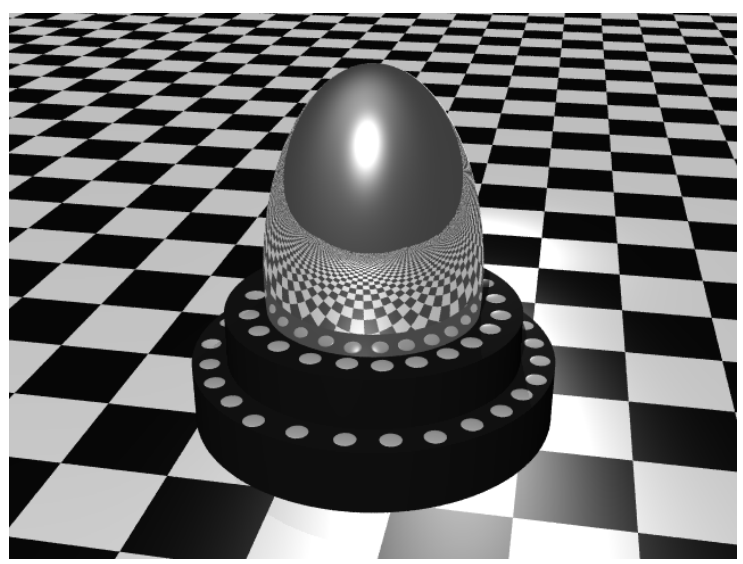

Figure 12. Example of new mirror design for improved catadioptric sensors without black needle, the two calibration circles appear on the bottom of the mirror, where calibration points can easily be retrieved.

\subsection{Improved Sensors}

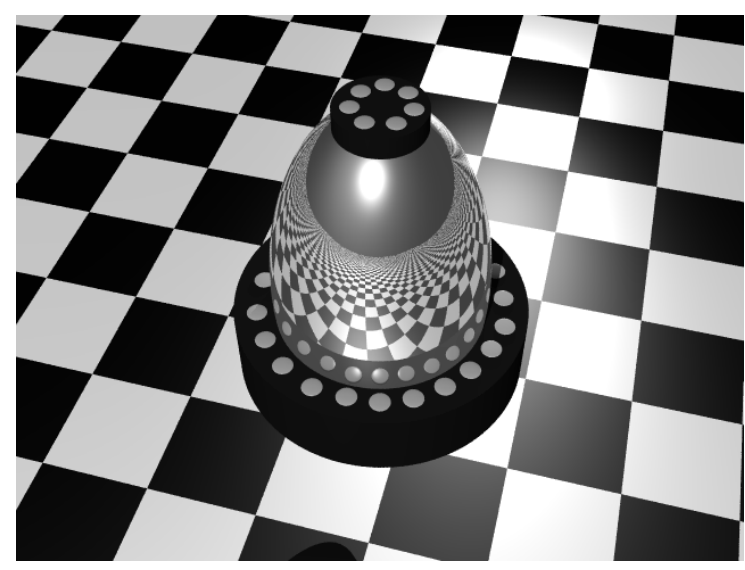

Figure 13. Example of new mirror design for improved catadioptric sensors without black needle with better calibration accuracy than previous examples.

Notice that the proposed catadioptric calibration method can be extended to new kinds of catadioptric sensors. This provides the possibility of manufacturing as shown by Fig. 11, Fig. 12 and Fig. 13 a new generation of sensors where two thin circles are positioned at two different heights. Calibration points are easy to detect and can also be added along these edges in such a way that the proposed processing is simplified. With these added features, the catadioptric sensor can be continuously calibrated, in real time.

Fig. 11 shows a mirror containing a black needle useful in the elimination of multiple reflections. Fig. 12 is an extension of the concept without the use of the black needle. Even if ellipses radius are larger, this configuration could suffer from a lack of precision due to two calibration plans $P_{1}$ and $P_{2}$ not far enough. Indeed, due to errors during calibration process, focal point $F^{\prime}$ is not estimated at its true position $F$. It is easy to show that the distance between $F$ and $F^{\prime}$ is decreasing with $d$, the distance between plans $P_{1}$ and $P_{2}$. As a consequence, to get a good precision, the best configuration is to use a pattern at the top and bottom of the mirror as shown in Fig. 13. Contrary to the first configuration, top calibration points are easier to detect, and thus allow to improve the calibration accuracy.

This improved sensors can be particularly useful in outdoor robotics applications where vibrations could misalign the camera and the mirror. 


\section{Conclusion}

We have developed a new method to calibrate panoramic catadioptric sensors. The main idea is to use the mirror boundaries like a calibration pattern for computing the intrinsic and extrinsic parameters of the CCD camera with respect to the mirror. The proposed algorithm is very fast and the calibration process is very simple to handle and can be applied on most catadioptric sensors unless the parameters of the mirror surface are known. The power of our method is its total autonomy: no calibration pattern in the scene, no features extraction in the image scene are required. With little modifications on the catadioptric sensor, no human intervention is needed and the process can be done any time required, even in real time to tackle the problems due to vibrations in mobile robot applications.

\section{Acknowledgments}

We would like to thank Vstone corporation [3] and Professor Hiroshi Ishiguro, from the Intelligent Robotics Laboratory - Department of Computer and Communication Sciences - Wakayama University, for providing us the catadioptric sensors used here.

\section{References}

[1] S. Baker and S. Nayar. A theory of single-viewpoint catadioptric image formation. International Journal of Computer Vision, 35(2):175-196, November 1999.

[2] C. Cauchois, E. Brassart, L. Delahoche, and T. Delhommelle. Reconstruction with the calibrated syclop sensor. In IEEE International Conference on Intelligent Robots and Systems (IROS'00), pages 1493-1498, Takamatsu, Japan, 2000.

[3] V. Corporation. http://www.vstone.co.jp.

[4] C. Geyer and K. Daniilidis. Para-catadioptric calibration. to appear in IEEE Trans. Pattern Analysis and Machine Intelligence, 2002.

[5] K. Gremban, C. H. Thorpe, and T. Kanade. Geometric camera calibration using systems of linear equations. IEEE Trans. Pattern Analysis and Machine Intelligence, pages 562-567, 1988.

[6] S. Kang. Catadioptric self-calibration. In IEEE Computer Vision and Pattern Recognition, pages I:201-207, Hilton Head Island, SC, 2000.

[7] H. A. Martins, J. Birk, and R. B. Kelley. Camera models based on data from two uncalibrated planes. Computer Graphics and Image Processing, 17:173-180, 1981.

[8] M. Pilou, A. Fitzgibbon, and R. Fisher. Ellipse specific direct least squre fitting. IEEE Int Conf on Image processing, 1996.

[9] T. Svoboda, T. Pajdla, and V. Hlaváč. Central panoramic cameras: Geometry and design. Research report
K335/97/147, Czech Technical University, Faculty of Electrical Engineering, Center for Machine Perception, FEL ČVUT, Karlovo náměstí 13, Praha, Czech Republic, December 1997.

[10] R. Swaminathan, M. D. Grossberg, and N. S. K. Caustics of catadioptric cameras. In IEEE International Conference on Computer Vision, Vancouver, Canada, 2001. 